\title{
Article
}

\section{Experimental Setup for Irradiation of Cell Cultures at L2A2}

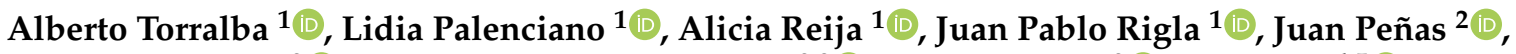 \\ Juan José Llerena ${ }^{2}{ }^{(0}$, Ramiro Contreras-Martínez ${ }^{2,3}{ }^{(\mathbb{D}}$, José Benlliure ${ }^{2}{ }^{(0)}$, Ana Vega ${ }^{4,5}{ }^{(0}$, \\ Miguel Elías Aguado-Barrera ${ }^{4,5}{ }^{\circ}$, Camilo Ruiz $^{3}{ }^{-1}$ and Michael Seimetz ${ }^{1, *} \mathbb{C}$
}

Citation: Torralba, A.; Palenciano, L.; Reija, A.; Rigla, J.P.; Peñas, J.; Llerena, J.J.; Contreras-Martínez, R.; Benlliure, J.; Vega, A.; Aguado-Barrera, M.E.; et al. Experimental Setup for Irradiation of Cell Cultures at L2A2. Quantum Beam Sci. 2022, 6, 10. https://doi.org/10.3390/qubs6010010 Academic Editor: Francesco Schillaci

Received: 23 December 2021

Accepted: 4 February 2022

Published: 21 February 2022

Publisher's Note: MDPI stays neutral with regard to jurisdictional claims in published maps and institutional affiliations.

Copyright: (C) 2022 by the authors. Licensee MDPI, Basel, Switzerland. This article is an open access article distributed under the terms and conditions of the Creative Commons Attribution (CC BY) license (https:// creativecommons.org/licenses/by/ $4.0 /)$.
1 Instituto de Instrumentación para Imagen Molecular (I3M), CSIC-Universitat Politècnica de València, Camino de Vera s/n, Ed. 8B-N-1, 46022 Valencia, Spain; ttalbertott@gmail.com (A.T.); lpalenciano@i3m.upv.es (L.P.); areivec@i3m.upv.es (A.R.); jprigla@i3m.upv.es (J.P.R.)

2 Universidade de Santiago de Compostela, 15782 Santiago de Compostela, Spain; juan.penas@usc.es (J.P.); juanjose.llerena@usc.es (J.J.L.); ramiro.contreras.martinez@usc.es (R.C.-M.); j.benlliure@usc.es (J.B.)

3 Instituto de Física Fundamental y Matemáticas, Universidad de Salamanca, Casas del Parque, 37008 Salamanca, Spain; camilo@usal.es

4 Fundación Pública Galega Medicina Xenómica, Hospital Clínico Universitario, Rúa da Choupana s/n, 15706 Santiago de Compostela, Spain; ana.vega@usc.es (A.V.); miguelelias.aguado@usc.es (M.E.A.-B.)

5 Instituto de Investigación Sanitaria de Santiago de Compostela (IDIS), Hospital Clínico Universitario, Travesía de Choupana s/n, 15706 Santiago de Compostela, Spain

* Correspondence: mseimetz@i3m.upv.es

\begin{abstract}
Laser-plasma proton sources and their applications to preclinical research has become a very active field of research in recent years. In addition to their small dimensions as compared to classical ion accelerators, they offer the possibility to study the biological effects of ultra-short particle bunches and the correspondingly high dose rates. We report on the design of an experimental setup for the irradiation of cell cultures at the L2A2 laboratory at the University of Santiago de Compostela, making use of a $1.2 \mathrm{~J} \mathrm{Ti}$ : Sapphire laser with a $10 \mathrm{~Hz}$ repetition rate. Our setup comprises a proton energy separator consisting of two antiparallel magnetic fields realized by a set of permanent magnets. It allows for selecting a narrow energy window around an adaptable design value of $5 \mathrm{MeV}$ out of the initially broad spectrum typical for Target Normal Sheath Acceleration (TNSA). At the same time, unwanted electrons and X-rays are segregated from the protons. This part of the setup is located inside the target vessel of the L2A2 laser. A subsequent vacuum flange sealed with a thin kapton window allows for particle passage to external sample irradiation. A combination of passive detector materials and real-time monitors is applied for measurement of the deposited radiation dose. A critical point of this interdisciplinary project is the manipulation of biological samples under well-controlled, sterile conditions. Cell cultures are prepared in sealed flasks with an ultra-thin entrance window and analysed at the nearby Fundación Pública Galega Medicina Xenómica and IDIS. The first trials will be centred at the quantification of DNA double-strand breaks as a function of radiation dose.
\end{abstract}

Keywords: laser-plasma interaction; TNSA; in vitro irradiation; dose measurement

\section{Introduction}

Radiation therapy with heavy particles (protons and ions) is an increasingly important modality of cancer management. Its clinical implementation has been accompanied by numerous in vitro experiments to investigate details of the cellular response to different radiation qualities and to measure the parameters of treatment models such as the relative biological effectiveness (RBE) [1,2]. Related research is partly carried out at particle therapy centres, some of which are equipped with dedicated test stages [3]. Several non-clinical accelerator facilities operating at high [4] or comparatively lower ion energies [5-8] have also implemented beamlines for the controlled irradiation of biological samples. Differences in cell response to radiation at clinical or ultra-high dose rates have received special 
attention [9]. The focus of the present research on FLASH radiotherapy [10] underlines the relevance of dose-rate effects for the improvement of cancer treatment.

Proton and ion accelerators based on ultra-short laser-plasma interactions have received much attention for their potential applications in medicine and biomedical research [11]. The use of laser-ion sources in particle therapy is not straightforward, as the ion energies are typically distributed over a wide spectral range and the maximum energies presently tend to be far below clinical standards. At first sight, the irradiation of in vitro samples, initiated in 2009 by A. Yogo et al. [12], might be considered not more than an intermediate step until laser-based facilities reach the same level of maturity as the radiofrequency cyclotrons and synchrotrons of modern therapy facilities. Nevertheless, during recent years, the intrinsically very short (nanosecond) particle bunch times from laser-plasma accelerators have been recognised as an outstanding tool for the study of biological effects at ultra-high dose rates. Several laser laboratories have implemented dedicated setups for the controlled irradiation of cell cultures (for a recent review, see [13]). The feasibility of related experiments with higher organisms such as zebrafish embryos [14] and the uniform dose deposition in volumes of the size of mouse tumour models [15] have been demonstrated as well.

A laser-based setup for the irradiation of biological samples requires a few key components, the first one being a stable ion accelerator. Some of the previously reported cell culture experiments were performed at Ti:Sapphire laser facilities with pulse durations of $\sim 30 \mathrm{fs}$ and energies $\leq 3$ J on target [12,16-20]. The accelerated particles (protons) had energies ranging from 2.1 to $15 \mathrm{MeV}$, which is sufficient to traverse a thin vacuum window and reach the cell samples which were maintained at atmospheric pressure. In order to measure the applied dose, the energies of the protons at the sample surface and the particle fluence have to be known. This generally implies the necessity for selecting a narrow interval out of the initially broad ion spectra and for the implementation of suitable detectors.

We have recently developed an experimental setup for cell culture irradiation at the Laser Laboratory for Acceleration and Medical Applications (L2A2) at Santiago de Compostela, making use of the $1.2 \mathrm{~J} / 35 \mathrm{fs}$ Ti: Sapphire beam line. A wheel-like, thinfoil target assembly with active position control and the capacity to perform more than 2000 shots at a $10 \mathrm{~Hz}$ repetition rate has previously been implemented [21]. A combination of two antiparallel magnetic fields allows for selecting protons with narrow energy spread (Section 2). We have performed simulations to estimate the impact of geometrical details on the particle fluence and the related dose per shot. Outside the vacuum system, various detectors can be applied to measure the particle fluence (Section 3). The preparation of cell cultures at an external microbiology laboratory as well as their handling under sterile conditions will be briefly presented in Section 4.

\section{Energy Selector}

Unlike with classical (rf) accelerators, the energies of particles released from ultrashort laser-plasma interactions are usually not concentrated in narrow intervals. Under the experimental conditions given at L2A2 in terms of focussed intensities $I_{L} \simeq 10^{19} \mathrm{~W} / \mathrm{cm}^{2}$ and pulse contrast $C=10^{-10}$ impinging on micrometer-thin target foils, the dominating mechanism for the generation of energetic protons is Target Normal Sheath Acceleration (TNSA) [22]. The resulting ion spectra have $100 \%$ spread up to a maximum energy of a few $\mathrm{MeV}$, with particle numbers per $\mathrm{MeV}$ strongly decreasing from low to high energies. For cell culture irradiation, a known radiation dose is to be applied to biological samples, and all the protons should ideally have similar linear energy transfer (LET). Therefore, it is necessary to select particles only from a limited spectral range. This can be achieved by spatial separation of the ions in a set of magnetic fields. In related experiments, different field geometries have been applied, comprising a single dipole field perpendicular to the trajectories [16,23-25] or a sequence of two antiparallel fields [12,19]. These cause a lateral displacement of the particle beams; longer chicanes can be used to guide the ions back to their initial axis [26]. Combinations of quadrupole magnets may be adequate for 
increasing the particle densities within some spectral range; however, it compromises the beam uniformity within the irradiated area $[17,27]$.

We have opted for a combination of two antiparallel dipole fields generated by permanent C-magnets, as shown in Figure 1. This assembly comprises two sets of five C-magnets each, with the individual magnets made of two neodymium blocks mounted on a stainless steel structure; the yokes are nickel coated for improved vacuum compatibility. The maximum field inside the gap of a single C-magnet is about $B_{y}=0.63 \mathrm{~T}$. The three field components of a single magnet have been simulated with COMSOL Multiphysics. In addition, the main component, $B_{y}$, has been measured throughout a mesh of positions on the central plane. The numerical values from the simulation have been scaled to the experimental results to obtain a 3D field map. Each magnet is mounted between a pair of rails (the top rails are omitted in Figure 1) to maintain a predefined distance between neighbouring blocks and to freely adjust the lateral positions. Thus, the entire geometry can be adapted to the energies of the proton beam or to other ion species and charge states, e.g., carbon ions.

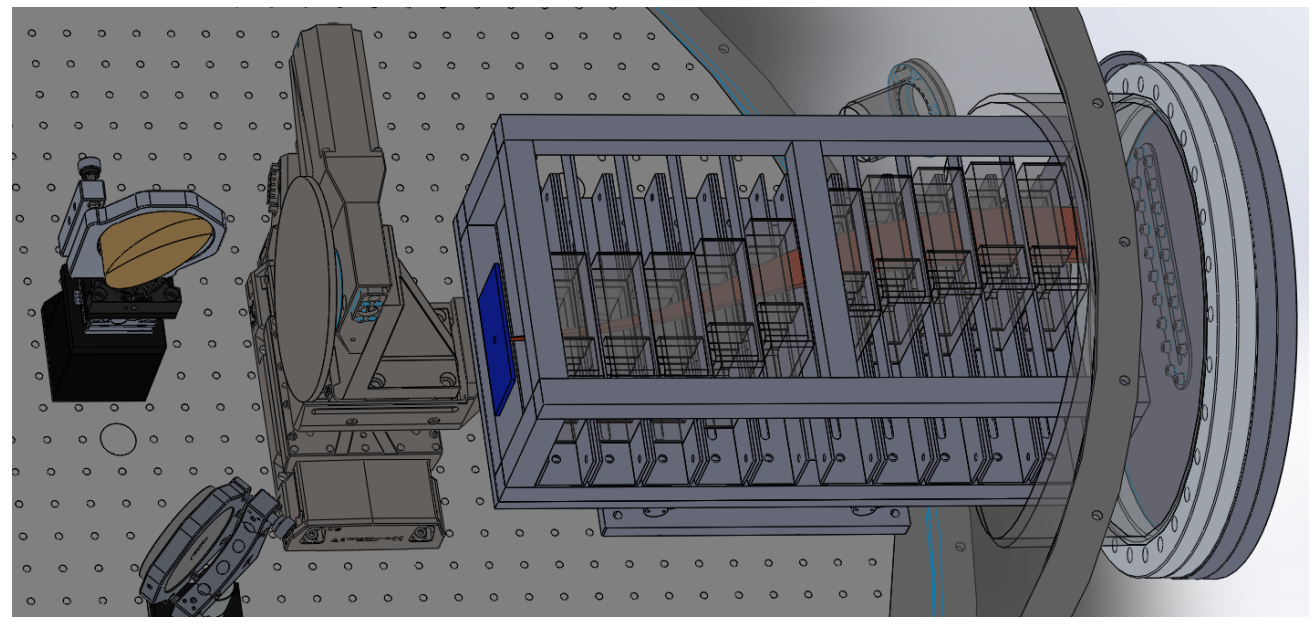

Figure 1. Energy selector made of permanent magnets inside the L2A2 target chamber. The pinhole collimator is highlighted in blue; proton trajectories are sketched in red.

We have calculated the trajectories of protons to study the influence of various geometrical factors of the energy selector on the particle fluence at the vacuum exit flange and the single-shot dose on the cell samples. A typical spectral distribution of laser-accelerated protons [28] has been used to model the flux directly behind the target, assuming that all the particles of a single laser shot are concentrated in a cone of $20^{\circ}$ opening angle. A pinhole collimator with radius $r_{p}=0.5 \mathrm{~mm}$ at the entrance of the selector, at a distance $L_{p}$ from the laser target, determines the angular spread of the incoming beam. The trajectories of those particles that pass the collimator are traced with a Python code comprising a 3D combination of the magnetic fields of ten individual blocks. Inside the field volumes, the particle position and the velocity vector under influence of the Lorentz force are calculated with a fixed step size of 0.01 ns. For illustration, some trajectories of selected energies are plotted in Figure 2a for a given configuration of magnet positions. One detail that can be observed in Figure $2 b$, showing the corresponding hit coordinates on the exit plane, is a small displacement towards negative $y$ coordinates, which is due to the non-zero fringe fields $B_{x}, B_{z}$. Protons of $2 \mathrm{MeV}$ or less fall far from the centres of the second group of magnets and leave the selector at large deflection angles. Behind the last dipole, those protons of $\geq 3 \mathrm{MeV}$ are redirected approximately parallel to the longitudinal $(z)$ axis. The central hit positions of protons with 3, 4, or $5 \mathrm{MeV}$ are spatially separated. At $\geq 6 \mathrm{MeV}$, the angular spread of particles passing through the collimator results in overlapping spots far from the laser target. 

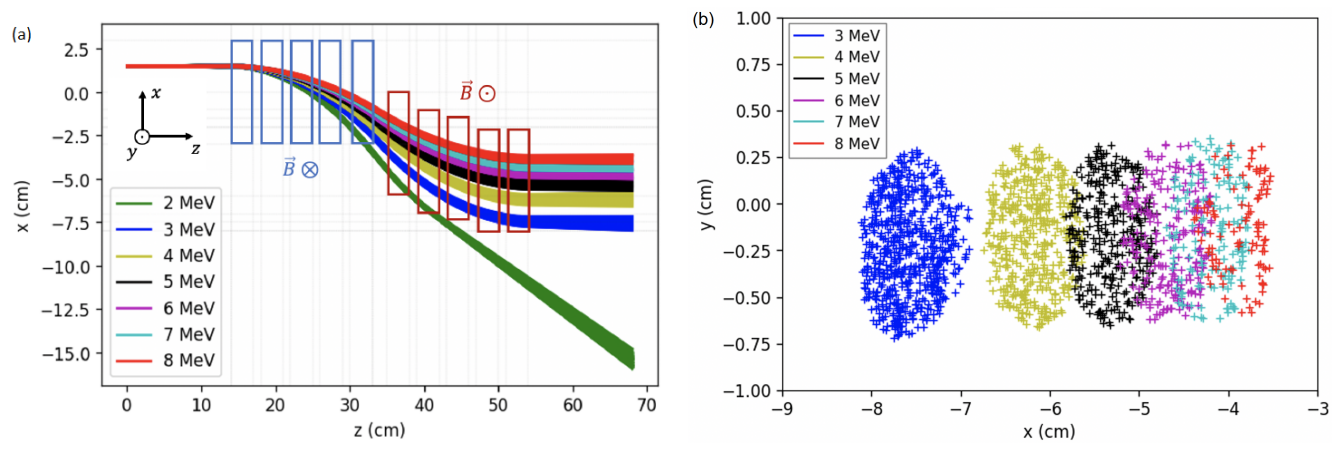

Figure 2. (a) Trajectories of protons with selected energies launched within the opening angle of the pinhole collimator, here at $L_{p}=70 \mathrm{~mm}$. Rectangles indicate the positions of neodymium blocks. (b) Hit positions of protons on exit plane.

We have further calculated the spectral distributions of protons incident on the irradiated cells in order to obtain the deposited dose. Here, particles released on the laser target cover a broad spectrum [28] as opposed to the discrete energy levels of Figure 2. Table 1 summarizes some of the results for the particle fluence on the cell surface, $\Phi$, the energy interval covered by these particles, and the total dose per shot, $D_{t}$, under variation of the collimator distance, with other geometric parameters kept constant. The energy loss in the kapton window and the PE foil of the culture dish has been taken into account. The lateral position of an irradiated area of $5 \times 5 \mathrm{~mm}^{2}$ is chosen in a way that protons of a predefined energy, $E_{\mathcal{C}}$, with initial momentum in the target normal direction hit the centre of this spot. The spectral distribution of these particles for one such configuration is shown in Figure 3a. We obtain proton fluences of the order 1.6-4.1 $\times 10^{6} / \mathrm{cm}^{2}$ and dose values of 18.0-64.7 mGy. For comparison, in related publications single-shot fluences between $1.5 \times 10^{6} / \mathrm{cm}^{2}$ and $3.3 \times 10^{8} / \mathrm{cm}^{2}$ on the sample surface were presented $[12,17,20,26]$. For the dose from a single laser shot, values $\leq 1 \mathrm{~Gy}$ (the lowest one being $0.081 \mathrm{~Gy}$ ) have been reported from various experiments $[12,16,18,19,26,29,30]$ while others were in excess of $1 \mathrm{~Gy} /$ shot $[14,17,20,23-25]$. In our setup, the single-shot fluence and the corresponding dose may be increased by choice of a larger solid angle, either through the distance or the radius of the collimator. However, the energy spread of the protons increases at the same time, and thus, the relative error of the radiation dose increases as well.

With a lower magnetic field or a smaller number of $C$ magnets, the lateral separation of particles would be less pronounced. As a consequence, the fluence on the target would increase at the cost of higher dose uncertainties.

Table 1. Simulated particle numbers, energy intervals, and corresponding radiation doses on the cell sample for different pinhole parameters.

\begin{tabular}{cccc}
\hline $\boldsymbol{L}_{\boldsymbol{p}} \mathbf{( \mathbf { m m } )}$ & $\boldsymbol{\Phi}\left(\mathbf{c m}^{-2}\right)$ & $E(\mathbf{M e V})$ & $\boldsymbol{D}_{t} \pm \Delta \boldsymbol{D}(\mathbf{m G y})$ \\
\hline$E_{c}=4 \mathrm{MeV}:$ & & & \\
50 & $4.1 \times 10^{6}$ & $2.9-4.9$ & $64.7 \pm 5.9$ \\
70 & $2.6 \times 10^{6}$ & $3.1-4.7$ & $40.7 \pm 2.8$ \\
100 & $1.8 \times 10^{6}$ & $3.3-4.4$ & $28.9 \pm 1.5$ \\
\hline$E_{\mathcal{c}}=5 \mathrm{MeV}:$ & & & \\
50 & $3.9 \times 10^{6}$ & $3.7-6.4$ & $51.6 \pm 5.3$ \\
70 & $2.8 \times 10^{6}$ & $3.8-6.1$ & $37.4 \pm 3.0$ \\
100 & $1.9 \times 10^{6}$ & $4.0-5.8$ & $24.5 \pm 1.4$ \\
\hline$E_{\mathcal{c}}=6 \mathrm{MeV}:$ & & & \\
50 & $3.3 \times 10^{6}$ & $4.3-8.1$ & $37.1 \pm 4.2$ \\
70 & $2.3 \times 10^{6}$ & $4.7-7.6$ & $26.0 \pm 2.2$ \\
100 & $1.6 \times 10^{6}$ & $5.0-7.2$ & $18.0 \pm 1.2$ \\
\hline
\end{tabular}



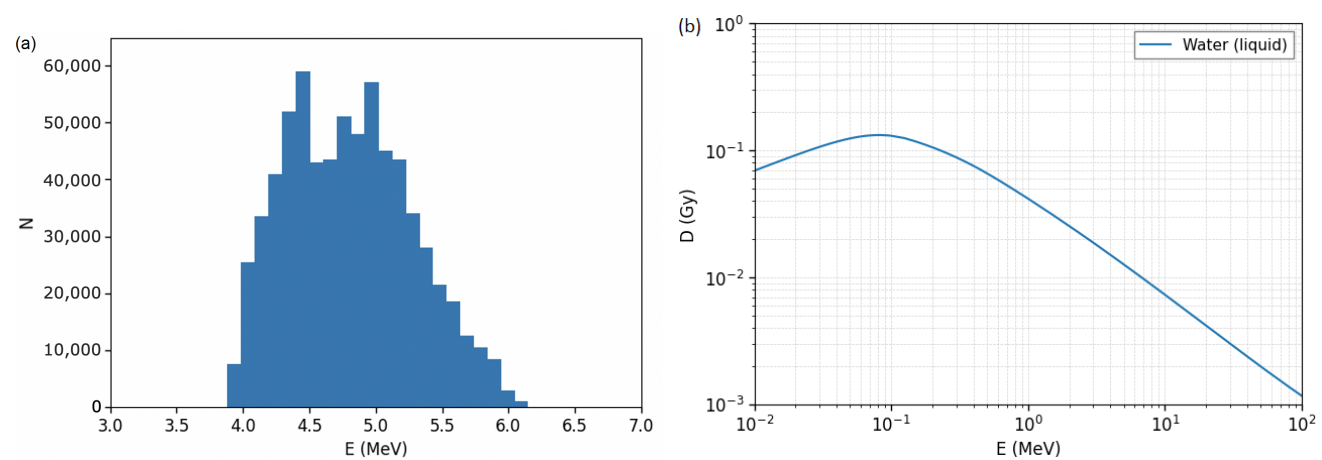

Figure 3. (a) Spectral distribution of protons in a $0.25 \mathrm{~cm}^{2}$ area at the surface of the cell culture dish for one of the simulated configurations $\left(L_{p}=70 \mathrm{~mm}, E_{c}=5 \mathrm{MeV}\right)$. (b) Correlation between proton energy and dose deposition in water for a fluence $\Phi=10^{6} / \mathrm{cm}^{2}$.

In Table $1, D_{t}$ refers to the total radiation dose in water. It is calculated from the linear energy transfer (LET) in water, $d E / d x$, the density, $\rho=1 \mathrm{~g} / \mathrm{cm}^{3}$, and the fluence via

$$
D=\frac{d E}{d x} \frac{1}{\rho} \Phi=\frac{d E}{d x}\left[\frac{\mathrm{keV}}{\mu \mathrm{m}}\right] \cdot \Phi\left[\frac{1}{\mathrm{~cm}^{2}}\right] \cdot 1.602 \times 10^{-9} \mathrm{~Gy} .
$$

LET values have been obtained from the NIST database [31]. The corresponding doses for $\Phi=10^{6} / \mathrm{cm}^{2}$ are shown as a function of particle energies in Figure $3 \mathrm{~b}$. For protons spread over a range of energies, such as in Figure $3 a$, the mean dose per particle is given by

$$
D_{m}=\frac{1}{N} \sum_{i} n\left(E_{i}\right) D\left(E_{i}\right), N=\sum_{i} n\left(E_{i}\right)
$$

with variance

$$
\sigma_{D}^{2}=\frac{1}{N} \sum_{i} n\left(E_{i}\right)\left(D\left(E_{i}\right)-D_{m}\right)^{2}
$$

summing over energy bins $E_{i}$. The total dose applied to the irradiated cells is $D_{t}=N D_{m}$ with uncertainty $\Delta D=D_{t} \sigma_{D} / D_{m}$. For the spectral distribution of Figure $3 a$, the relative uncertainty amounts to $\Delta D / D=8.0 \%$; the values for all listed configurations range from $5.2 \%$ to $11.3 \%$. This dose error is only due to the finite geometrical acceptance assuming that all laser shots produce the same particle spectra. In practice, significant shot-to-shot fluctuations must be expected, and the dominant factor of the dose variability may stem from the measured fluence. For comparison, for single laser shots, values of $\Delta D / D$ between $6.5 \%$ and $12.7 \%$ were reported in [23], about $15 \%$ were reported in [25], and about $23 \%$ were reported in $[19,30]$.

\section{Particle Fluence Detectors}

In order to determine the dose deposition on the irradiated cell samples, the particle fluence must be precisely known. For laser-plasma accelerators, this task is especially difficult due to the nonlinear response of electronic detectors to ions concentrated in ultrashort bunches. In addition, shot-to-shot fluctuations must be considered a major source of uncertainty. Contrary to particle therapy facilities working at $10 \mathrm{~s}$ or $100 \mathrm{~s}$ of $\mathrm{MeV}$, for laserbased sources, an additional challenge is imposed by the typically modest proton energies of a few MeV. The range of these particles in solid matter is very limited, and spectral shifts due to the energy loss even when traversing a layer of a few microns thickness have to be taken into account for dose calculation. Our setup comprises a combination of passive detector materials and electronic devices to be used for online monitoring, the measurement of accumulated dose, and cross-calibration. Measuring the fluence behind the irradiated specimen is currently not feasible at the $1.2 \mathrm{~J}$ laser line of L2A2 because protons of $\leq 10 \mathrm{MeV}$ 
are stopped in the cell culture flasks (see Section 4), and still, higher energies are not likely to be achieved according to TNSA scaling models.

In order to study the influence of transmission detectors on the particle spectra, we have calculated the energy losses in corresponding material layers (Figure 4) using the database SRIM [32]. The coloured lines represent selected energies within an initially broad spectrum up to $10 \mathrm{MeV}$ inside the vacuum system (labelled "incoming"). Protons pass through a vacuum window sealed with a $12.8 \mu \mathrm{m}$ thick kapton foil. In addition, they cross a short distance in air and a $7 \mu \mathrm{m}$ thick polyethylene foil, which seals the culture flask before hitting the actual cells. The resulting spectrum for this case is labelled "Kapton + PE"; colours correspond to the original levels. Detectors used in transmission mode in front of the specimen will further reduce the final energies, as discussed below.

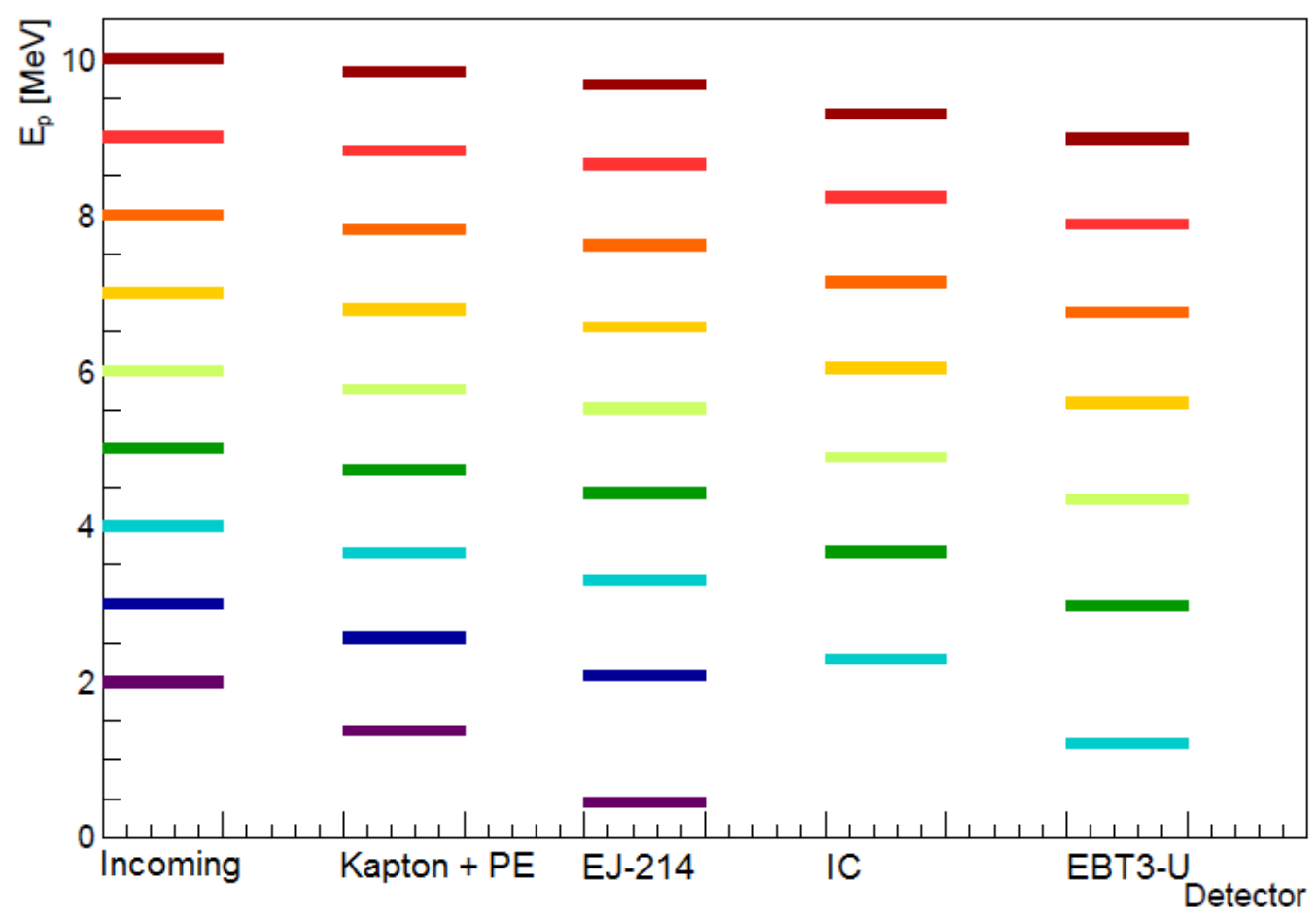

Figure 4. Particle energies after passage through fluence detectors. Colours correspond to the selected lines of the incoming beam in vacuum.

Solid-state nuclear track detectors made of PADC (polyallyl-diglycol-carbonate, also denominated CR-39) allow for a very precise determination of incident ion numbers in combination with a highly specific reponse to heavy particles. This transparent resin is typically manufactured in plates of $0.2-1 \mathrm{~mm}$ thickness. PADC has been applied in related experiments for fluence measurements either in close proximity to the cell cultures during sample irradiation [12] or for the control of shot-to-shot fluctuations and crosscalibration [16,26,29]. We use a special brand of material (Radosys RS39) where proton tracks, after $2 \mathrm{~h}$ etching in $6.25 \mathrm{~N} \mathrm{NaOH}$ at $90{ }^{\circ} \mathrm{C}$, have diameters of $4-8 \mu \mathrm{m}$, and their size does not vary strongly with the particle energy [33]. With automatic image analysis methods, track densities up to $1-2 \times 10^{6} / \mathrm{cm}^{2}$ can be determined.

Radiochromic film (RCF) is another kind of passive detector frequently used in radiobiology experiments with laser-accelerated protons [17,24,25]. Standard types for clinical applications (Gafchromic EBT2, EBT3) consist of a sensitive layer sandwiched between two protective polyester films. A special version, EBT3-U ("unlaminated"), with only one polyester substrate and the sensitive layer on top, is more suitable for short-range particles such as carbon ions of a few $\mathrm{MeV} / u$ [34]. Its dynamic range (0.1-20 Gy) covers a major part of the dose intervals relevant for cell irradiations. With a total thickness of $153 \mu \mathrm{m}$, EBT3-U absorbs more than $1 \mathrm{MeV}$ of kinetic energy from incident protons of $\leq 8 \mathrm{MeV}$ (Figure 4 , 
"EBT3-U"; the energy losses in the kapton and PE foils have been taken into account). Protons with less than $\sim 4 \mathrm{MeV}$ in vacuum will be stopped before hitting the cells. Another brand of RCF with an unprotected sensitive layer, Gafchromic HD-V2, is only $109 \mu \mathrm{m}$ thin, but its dynamic range covers higher radiation doses of the order 10-1000 Gy [35]. Therefore, we do not aim at applying these RCF in transmission mode in front of the specimen at L2A2. Nevertheless, they will be used for cross-calibration purposes.

Ionization chambers (IC) are widely used as beam monitors for clinical accelerators. In the context of radiobiological research with laser-accelerated protons, a specially developed dosimetric system with a custom-made transmission IC has been reported [36]. It comprises three metalized kapton foils of $7.5 \mu \mathrm{m}$ thickness each. The commercial IC model PTW 786, with three graphite-coated kapton foils of $25 \mu \mathrm{m}$ thickness each, has been applied in related experiments [30] after calibration at a clinical facility [20]. We have chosen the PTW 786 as part of our setup at L2A2 as well. For protons of 6-8 MeV, the calculated energy loss upon transmission is about $1 \mathrm{MeV}$ (Figure 4, "IC"). For continuous particle beams, the charge output of ionization chambers is proportional to the beam charge. For pulsed beams, to the contrary, deviations from this linear response have been observed [37]. Therefore, we aim at calibrating the PTW 786 with laser-accelerated protons against passive materials (PADC and EBT3-U).

As an alternative with significantly lower area density, we have developed an ion detector based on an ultra-thin plastic scintillator of $25 \mu \mathrm{m}$ thickness (Eljen EJ-214). This free-standing sheet is coupled to a low-noise photomultiplier tube (PMT). Our preliminary tests have confirmed the capacity of this device to detect $\alpha$ and $\beta$ particles from radioactive sources. Here, too, the response to ultra-short proton bunches and the relation between particle numbers and PMT anode pulses will be calibrated at L2A2. Output signals are recorded on a fast oscilloscope. This device is intended as shot-to-shot transmission monitor of the particle fluence as protons passing through the scintillator lose only $\sim 0.5 \mathrm{MeV}$ for initial energies $\geq 5 \mathrm{MeV}$ (Figure 4, "EJ-214").

\section{Cell Cultures}

Experimental studies dedicated to radiobiological effects of ionizing radiation do not only require precise knowledge of the particle spectra and fluence as well as the total dose on the irradiated specimen. In addition, delicate samples must be kept under well-controlled, sterile conditions. For experiments at L2A2, cell cultures are grown in commercial flasks (TPP, $25 \mathrm{~cm}^{2}$ ) which on one side are sealed with a $7 \mu \mathrm{m}$ thick polyethylene foil. Cells with typical diameters around $15 \mu \mathrm{m}$ form a stable monolayer on this peel-off foil previously treated with fibronectin. Only for the time of irradiation, the flask is kept in vertical position. For protons of a few $\mathrm{MeV}$, the energy loss inside the foil is almost negligible. The rear and side walls of the flask, to the contrary, are made of polystyrene of $1 \mathrm{~mm}$ thickness. Protons of $<10 \mathrm{MeV}$ are stopped inside this material; thus, it is not possible to measure their fluence behind the sample.

A sufficient number of cells is seeded to guarantee a confluency of $90 \%$ at the time of irradiation. On an irradiated surface of $5 \times 5 \mathrm{~mm}^{2}$, we expect about $10^{5}$ cells, which is adequate to perform different types of analyses to evaluate biological effects. For example, the use of immunofluorescence to detect DNA double-strand breaks, the study of cell viability, and the quantification of cell death after exposure require only around 100 cells per field. An even smaller number of cells is needed for colony formation experiments (2-50 cells $\left./ \mathrm{cm}^{2}\right)$. In contrast, analyses of protein activation based on Western blots [30] require several million cells, which is more than we can presently obtain from a single irradiation procedure.

Cell cultures are grown and analysed post-exposure in the Functional Genomics lab at the Fundación Pública Galega de Medicina Xenómica (FPGMX), which is located $1.5 \mathrm{~km}$ from L2A2. The entire process of transport and sample irradiation can be realized within one hour. During that time, except for a few minutes of radiation exposure, the flasks are stored at constant temperature inside a portable incubator. Sham-irradiated 
specimen allow for the control of adverse effects of this manipulation procedure. We have tested the sample preparation and displacement with human basal alveolar epithelial adenocarcinoma (A549) cells, which are widely used for radiobiological studies. In general, cancerous cells are frequently chosen as models for in vitro experiments due to the close relation to particle therapy. However, research on the response of healthy tissues to ionizing radiation is ongoing as well. The use of commercial cell lines facilitates the comparison of quantitative results from our experiments with published data, e.g., in terms of survival rates. Nevertheless, the radiation exposure of specific specimen, such as cell lines derived from cancer patients, can be equally realised with our setup.

In a uniform radiation field, monolayer cell cultures allow for the application of very similar dose to all the cells inside the irradiated area. This is especially important for incident particle energies of a few $\mathrm{MeV}$, which is close to the Bragg peak, where LET values vary significantly along the tracks inside matter, and the total range within the specimen is limited to some hundreds of micrometers. A wealth of in vitro models have been proposed for preclinical research with proton beams [38], some of which offer insight into intercellular interactions and allow for studying the radiation response of tissues rather than individual cells. We may extend the variety of specimen in our experiments towards thin-cut tumour slices and cancer organoids with about $500 \mu \mathrm{m}$ thickness. Such samples should be kept in a horizontal position during irradiation. This can be technically realized by implementing a $90^{\circ}$ bending magnet into our setup. Nevertheless, it must be previously demonstrated that proton energies around $10 \mathrm{MeV}$ at high fluence, which are required for a uniform, volumetric dose deposition, can be reached with the laser intensities available at L2A2. Due to the same limitation, we presently do not envisage in vivo studies with yet larger sample volumes.

\section{Conclusions and Outlook}

An experimental setup for the irradiation of cell cultures with laser-accelerated protons has been prepared at the $1.2 \mathrm{~J}$ pulsed laser beamline of L2A2. It comprises a pair of antiparallel magnetic dipole fields which allow for selecting a narrow range of particle energies from the initially broad spectral distribution typical for TNSA. The precise operational parameters are subject to the ongoing optimization of the laser-plasma interaction. Our simulations based on related results indicate that a single-shot dose of 18-65 mGy can be applied to an irradiated area of $5 \times 5 \mathrm{~mm}^{2}$. Throughout this area, the relative dose uncertainty due to the finite width of the incident particle spectrum is below $11 \%$. Protons are concentrated in pulses of about $10 \mathrm{~ns}$ duration, which is given by time-of-flight differences among their energies, with corresponding peak dose rates of the order $10^{6} \mathrm{~Gy} / \mathrm{s}$. Within a few minutes of continuous operation, our samples may receive an accumulated dose of several Gy. The total radiation dose will be determined with active and passive fluence monitors. A specially developed, ultra-thin scintillation detector is suitable for shot-to-shot measurements at a $10 \mathrm{~Hz}$ repetition rate. We have calculated the influence of particle passage through intermediate materials on their final energies. This includes the thin entrance window of the cell culture flasks.

In a commissioning phase, the actual proton spectra are to be determined. This will allow for fixing one central energy on the secondary target plane, and measuring the particle fluence at that position. Then, our aim is to perform systematic studies of biological effects of particle radiation on living cells. The influence of ultra-high dose rates may be assessed through comparison with protons of similar energies from classical accelerators [7]. The necessary tools for the observation of DNA repair dynamics (e.g., through the formation of $\gamma \mathrm{H} 2 \mathrm{AX}$ foci) or the measurement of cell proliferation as a function of the total dose are at hand. A typical experiment with a few samples for each of several scenarios may require the preparation, irradiation, and analysis of tens of specimen. The next big challenge for our group resides in finding the most promising cellular response pathways to investigate with our setup. 


\begin{abstract}
Author Contributions: Conceptualization, A.T. and M.S.; methodology, M.E.A.-B., A.V. and M.S.; software, A.T. and A.R.; validation, J.J.L., J.P. and C.R.; formal analysis, A.T. and A.R.; investigation, L.P., A.R., R.C.-M. and J.P.R.; resources, J.B., A.V. and M.S.; writing-original draft preparation, M.S.; writing-review and editing, J.P., J.B., A.V., M.E.A.-B. and C.R.; visualization, A.R. and M.S.; supervision, M.S.; project administration, M.S.; funding acquisition, J.B. and M.S. All authors have read and agreed to the published version of the manuscript.
\end{abstract}

Funding: Projects RTI2018-101578-B-C21 and RTI2018-101578-B-C22 are funded by MCIN/AEI /10.13039/501100011033 and by FEDER “Una manera de hacer Europa”. Project AICO/2020/207 is funded by Generalitat Valenciana. Supports PEJ2018-002035-A and PEJ2018-002037-A are financed by AEI and "El FSE invierte en tu futuro". Additional funding is by FPI predoctoral grant BES-201708917 and Unidad de Excelencia María de Maeztu, MdM-2016-0692-17-2. Action is co-financed by the European Union through the Programa Operativo del Fondo Europeo de Desarrollo Regional (FEDER) of the Comunitat Valenciana 2014-2020 (IDIFEDER/2018/022 and IDIFEDER/2021/004). FPGMXIDIS research is partially supported by the Spanish Instituto de Salud Carlos III (ISCIII) funding, which is an initiative of the Spanish Ministry of Economy and Innovation partially supported by European Regional Development FEDER Funds (INT20/00071, PI19/01424, AC18/00117) and through the Autonomous Government of Galicia (Consolidation and structuring program: IN607B) given to A. Vega.

Data Availability Statement: Data presented in this study are available on request from the corresponding author.

Conflicts of Interest: The authors declare no conflict of interest. The funders had no role in the design of the study; in the collection, analyses, or interpretation of data; in the writing of the manuscript, or in the decision to publish the results.

\title{
References
}

1. Friedrich, T.; Scholz, U.; Elsässer, T.; Durante, M.; Scholz, M. Systematic analysis of RBE and related quantities using a database of cell survival experiments with ion beam irradiation. J. Radiat. Res. 2012, 54, 494-514. [CrossRef] [PubMed]

2. Friedrich, T.; Pfuhl, T.; Scholz, M. Update of the particle irradiation data ensemble (PIDE) for cell survival. J. Radiat. Res. 2021, 62, 645-655. [CrossRef] [PubMed]

3. Henthorn, N.T.; Sokol, O.; Durante, M.; De Marzi, L.; Pouzoulet, F.; Miszczyk, J.; Olko, P.; Brandenburg, S.; van Goethem, M.J.; Barazzuol, L.; et al. Mapping the Future of Particle Radiobiology in Europe: The INSPIRE Project. Front. Phys. 2020, 8 , 565055. [CrossRef]

4. Patera, V.; Prezado, Y.; Azaiez, F.; Battistoni, G.; Bettoni, D.; Brandenburg, S.; Bugay, A.; Cuttone, G.; Dauvergne, D.; de France, G.; et al. Biomedical Research Programs at Present and Future High-Energy Particle Accelerators. Front. Phys. $2020,8,380$. [CrossRef] [PubMed]

5. Wéra, A.C.; Riquier, H.; Heuskin, A.C.; Michiels, C.; Lucas, S. In vitro irradiation station for broad beam radiobiological experiments. Nucl. Instrum. Methods Phys. Res. Sect. B Beam Interact. Mater. At. 2011, 269, 3120-3124. [CrossRef]

6. Manti, L.; Campajola, L.; Perozziello, F.M.; Kavanagh, J.N.; Schettino, G. Development of a low-energy particle irradiation facility for the study of the biological effectiveness of the ion track end. J. Phys. Conf. Ser. 2012, 373, 012019. [CrossRef]

7. Baratto-Roldán, A.; Jiménez-Ramos, M.d.C.; Jimeno, S.; Huertas, P.; García-López, J.; Gallardo, M.I.; Cortés-Giraldo, M.A.; Espino, J.M. Preparation of a radiobiology beam line at the $18 \mathrm{MeV}$ proton cyclotron facility at CNA. Phys. Medica Eur. J. Med Phys. 2020, 74, 19-29. [CrossRef]

8. Viñals, S.; Sánchez-Parcerisa, D.; Fraile, L.M.; España, S.; García, G.; García-Díaz, M.; SánchezTembleque, V.; Udías, J.M. Characterization of the proton pulsed beam at CMAM. EPJ Web Conf. 2021, 253, 04027. [CrossRef]

9. Auer, S.; Hable, V.; Greubel, C.; Drexler, G.A.; Schmid, T.E.; Belka, C.; Dollinger, G.; Friedl, A.A. Survival of tumor cells after proton irradiation with ultra-high dose rates. Radiat. Oncol. 2011, 6, 139. [CrossRef]

10. Hughes, J.R.; Parsons, J.L. FLASH Radiotherapy: Current Knowledge and Future Insights Using Proton-Beam Therapy. Int. J. Mol. Sci. 2020, 21, 6492. [CrossRef]

11. Ledingham, K.; Bolton, P.; Shikazono, N.; Ma, C.M. Towards Laser Driven Hadron Cancer Radiotherapy: A Review of Progress. Appl. Sci. 2014, 4, 402-443. [CrossRef]

12. Yogo, A.; Sato, K.; Nishikino, M.; Mori, M.; Teshima, T.; Numasaki, H.; Murakami, M.; Demizu, Y.; Akagi, S.; Nagayama, S.; et al. Application of laser-accelerated protons to the demonstration of DNA double-strand breaks in human cancer cells. Appl. Phys. Lett. 2009, 94, 181502. [CrossRef]

13. Chaudhary, P.; Milluzzo, G.; Ahmed, H.; Odlozilik, B.; McMurray, A.; Prise, K.M.; Borghesi, M. Radiobiology Experiments with Ultra-high Dose Rate Laser-Driven Protons: Methodology and State-of-the-Art. Front. Phys. 2021, 9, 75. [CrossRef]

14. Rösch, T.F.; Szabó, Z.; Haffa, D.; Bin, J.; Brunner, S.; Englbrecht, F.S.; Friedl, A.A.; Gao, Y.; Hartmann, J.; Hilz, P.; et al. A feasibility study of zebrafish embryo irradiation with laser-accelerated protons. Rev. Sci. Instrum. 2020, 91, 063303. [CrossRef] [PubMed] 
15. Brack, F.E.; Kroll, F.; Gaus, L.; Bernert, C.; Beyreuther, E.; Cowan, T.E.; Karsch, L.; Kraft, S.; Kunz-Schughart, L.A.; Lessmann, E.; et al. Spectral and spatial shaping of laser-driven proton beams using a pulsed high-field magnet beamline. Sci. Rep. 2020, 10, 9118. [CrossRef] [PubMed]

16. Kraft, S.D.; Richter, C.; Zeil, K.; Baumann, M.; Beyreuther, E.; Bock, S.; Bussmann, M.; Cowan, T.E.; Dammene, Y.; Enghardt, W.; et al. Dose-dependent biological damage of tumour cells by laser-accelerated proton beams. New J. Phys. 2010, 12, 085003 [CrossRef]

17. Bin, J.; Allinger, K.; Assmann, W.; Dollinger, G.; Drexler, G.A.; Friedl, A.A.; Habs, D.; Hilz, P.; Hoerlein, R.; Humble, N.; et al. A laser-driven nanosecond proton source for radiobiological studies. Appl. Phys. Lett. 2012, 101, 243701. [CrossRef]

18. Zeil, K.; Baumann, M.; Beyreuther, E.; Burris-Mog, T.; Cowan, T.E.; Enghardt, W.; Karsch, L.; Kraft, S.D.; Laschinsky, L.; Metzkes, J.; et al. Dose-controlled irradiation of cancer cells with laser-accelerated proton pulses. Appl. Phys. B 2013, 110, 437-444. [CrossRef]

19. Raschke, S.; Spickermann, S.; Toncian, T.; Swantusch, M.; Boeker, J.; Giesen, U.; Iliakis, G.; Willi, O.; Boege, F. Ultra-short laser-accelerated proton pulses have similar DNA-damaging effectiveness but produce less immediate nitroxidative stress than conventional proton beams. Sci. Rep. 2016, 6, 32441. [CrossRef]

20. Pommarel, L.; Vauzour, B.; Mégnin-Chanet, F.; Bayart, E.; Delmas, O.; Goudjil, F.; Nauraye, C.; Letellier, V.; Pouzoulet, F.; Schillaci, F.; et al. Spectral and spatial shaping of a laser-produced ion beam for radiation-biology experiments. Phys. Rev. Accel. Beams 2017, 20, 032801. [CrossRef]

21. Peñas, J.; Cortina-Gil, D.; Martín, L.; Ruiz, C.; Seimetz, M.; Benlliure, J. A Multi-Shot Wheel-Target Assembly for Laser-Plasma Proton Acceleration. High Power Laser Sci. Eng. 2021, submitted.

22. Roth, M.; Schollmeier, M. Ion Acceleration-Target Normal Sheath Acceleration. CERN Yellow Rep. $2016,1,231$.

23. Fiorini, F.; Kirby, D.; Borghesi, M.; Doria, D.; Jeynes, J.C.G.; Kakolee, K.F.; Kar, S.; Litt, S.K.; Kirkby, K.J.; Merchant, M.J.; et al. Dosimetry and spectral analysis of a radiobiological experiment using laser-driven proton beams. Phys. Med. Biol. 2011, 56, 6969-6982. [CrossRef] [PubMed]

24. Doria, D.; Kakolee, K.F.; Kar, S.; Litt, S.K.; Fiorini, F.; Ahmed, H.; Green, S.; Jeynes, J.C.G.; Kavanagh, J.; Kirby, D.; et al. Biological effectiveness on live cells of laser driven protons at dose rates exceeding $10^{9} \mathrm{~Gy} / \mathrm{s}$. AIP Adv. 2012, 2, 011209. [CrossRef]

25. Hanton, F.; Chaudhary, P.; Doria, D.; Gwynne, D.; Maiorino, C.; Scullion, C.; Ahmed, H.; Marshall, T.; Naughton, K.; Romagnani, L.and Kar, S.; et al. DNA DSB Repair Dynamics following Irradiation with Laser-Driven Protons at Ultra-High Dose Rates. Sci. Rep. 2019, 9, 4471. [CrossRef]

26. Yogo, A.; Maeda, T.; Hori, T.; Sakaki, H.; Ogura, K.; Nishiuchi, M.; Sagisaka, A.; Kiriyama, H.; Okada, H.; Kanazawa, S.; et al. Measurement of relative biological effectiveness of protons in human cancer cells using a laser-driven quasimonoenergetic proton beamline. Appl. Phys. Lett. 2011, 98, 053701. [CrossRef]

27. Schillaci, F.; Pommarel, L.; Romano, F.; Cuttone, G.; Costa, M.; Giove, D.; Maggiore, M.; Russo, A.; Scuderi, V.; Malka, V.; et al. Characterization of the ELIMED Permanent Magnets Quadrupole system prototype with laser-driven proton beams. J. Instrum. 2016, 11, T07005. [CrossRef]

28. Fritzler, S.; Malka, V.; Grillon, G.; Rousseau, J.P.; Burgy, F.; Lefebvre, E.; d'Humières, E.; McKenna, P.; Ledingham, K.W.D. Proton beams generated with high-intensity lasers: Applications to medical isotope production. Appl. Phys. Lett. 2003, 83, 3039-3041. [CrossRef]

29. Manti, L.; Perozziello, F.; Borghesi, M.; Candiano, G.; Chaudhary, P.; Cirrone, G.; Doria, D.; Gwynne, D.; Leanza, R.; Prise, K.M.; et al. The radiobiology of laser-driven particle beams: Focus on sub-lethal responses of normal human cells. J. Instrum. 2017, 12, C03084. [CrossRef]

30. Bayart, E.; Flacco, A.; Delmas, O.; Pommarel, L.; Levy, D.; Cavallone, M.; Megnin-Chanet, F.; Deutsch, E.; Malka, V. Fast dose fractionation using ultra-short laser accelerated proton pulses can increase cancer cell mortality, which relies on functional PARP1 protein. Sci. Rep. 2019, 9, 10132. [CrossRef]

31. Berger, M.J.; Coursey, J.S.; Zucker, M.A.; Chang, J. Stopping-power and range tables for electrons, protons, and helium ions. In NIST Standard Reference Database 124; NIST: Gaithersburg, MD, USA, 1998. [CrossRef]

32. Ziegler, J.F.; Ziegler, M.D.; Biersack, J.P. SRIM - The Stopping Range of Ions in Matter (2010); Nucl. Instrum. Meth. Phys. Res. Sect. B Beam Interact. Mater. At. 2010, 268, 1818-1823. [CrossRef]

33. Seimetz, M.; Peñas, J.; Llerena, J.; Benlliure, J.; García López, J.; Millán-Callado, M.; Benlloch, J. PADC nuclear track detector for ion spectroscopy in laser-plasma acceleration. Phys. Medica Eur. J. Med. Phys. 2020, 76, 72-76. [CrossRef] [PubMed]

34. Milluzzo, G.; Ahmed, H.; Romagnani, L.; Doria, D.; Chaudhary, P.; Maiorino, C.; McIlvenny, A.; McMurray, A.; Polin, K.; Katzir, Y.; et al. Dosimetry of laser-accelerated carbon ions for cell irradiation at ultra-high dose rate. J. Phys. Conf. Ser. 2020, 1596, 012038. [CrossRef]

35. Seimetz, M.; Bellido, P.; Soriano, A.; García López, J.; Jiménez-Ramos, M.C.; Fernández, B.; Conde, P.; Crespo, E.; González, A.J.; Hernández, L.; et al. Calibration and Performance Tests of Detectors for Laser-Accelerated Protons. IEEE Trans. Nucl. Sci. 2015, 62, 3216-3224. [CrossRef]

36. Richter, C.; Karsch, L.; Dammene, Y.; Kraft, S.D.; Metzkes, J.; Schramm, U.; Schürer, M.; Sobiella, M.; Weber, A.; Zeil, K.; et al. A dosimetric system for quantitative cell irradiation experiments with laser-accelerated protons. Phys. Med. Biol. 2011, 56, 1529-1543. [CrossRef] 
37. Di Martino, F.; Giannelli, M.; Traino, A.C.; Lazzeri, M. Ion recombination correction for very high dose-per-pulse high-energy electron beams. Med. Phys. 2005, 32, 2204-2210. [CrossRef]

38. Suckert, T.; Nexhipi, S.; Dietrich, A.; Koch, R.; Kunz-Schughart, L.A.; Bahn, E.; Beyreuther, E. Models for Translational Proton Radiobiology-From Bench to Bedside and Back. Cancers 2021, 13, 4216. [CrossRef] 